\title{
Duflo-Moore Operator for The Square-Integrable Representation of the 2-Dimensional Affine Lie Group
}

\author{
Edi Kurniadi $^{1}$, Nurul Gusriani ${ }^{2}$, Betty Subartini ${ }^{3}$ \\ ${ }^{1}$ Universitas Padjadjaran Bandung, edi.kurniadi@unpad.ac.id \\ ${ }^{2}$ Universitas Padjadjaran Bandung, nurul.gusriani@unpad.ac.id \\ ${ }^{3}$ Universitas Padjadjaran Bandung, betty.subartini@unpad.ac.id
}

doi: https://doi.org/10.15642/mantik.2020.6.2.114-122

\begin{abstract}
Abstrak. Dalam artikel ini, dipelajari representasi quasi-regular dan representasi unitar tak tereduksi grup Lie affine $\mathrm{Aff}^{+}(1)$ berdimensi dua. Pertama, diberikan bukti lengkap dari hasil kerja Fuhr tentang transformasi Fourier untuk representasi quasi-regular dari $\mathrm{Aff}^{+}(1)$. Kedua, ketika representasi dari grup Lie affine $\mathrm{Aff}^{+}(1)$ adalah square-integrable maka dihitung operator DufloMoore secara langsung tanpa menggunakan transformasi Fourier seperti dalam hasil Fuhr.
\end{abstract}

Kata kunci: Grup Lie affine; Operator Duflo-Moore; Representasi square-integrable.

\begin{abstract}
In this paper, we study the quasi-regular and the irreducible unitary representation of affine Lie group $\mathrm{Aff}^{+}(1)$ of dimension two. First, we prove a sharpening of Fuhr's work of Fourier transform of quasi-regular representation of $\mathrm{Aff}^{+}(1)$. The second, in such the representation of affine Lie group $\mathrm{Aff}^{+}(1)$ is square-integrable then we compute its Duflo-Moore operator instead of using Fourier transform as in Führ's work.
\end{abstract}

Keywords: Affine Lie group; Duflo-Moore operator; Square-integrable representation. 


\section{Introduction}

The current research about square-integrable representations of Lie groups can be found, for instance in [1] and [2]. In the previous work, the notion of square-integrable representation of a Lie group associating to wavelet transforms was introduced by Grossmann, Morlet, and Paul (see [3]). Particularly, they investigated the nice examples of a square-integrable representation of $a x+b$ - group, known as affine Lie group Aff(1) as can be seen in [4]. In the other hand, the research about $a x+b$-groups can also be found, for instance in [5] and [6].

It is well known that $\operatorname{Aff}(1)$ is the exponential solvable Lie group which is non unimodular group whose Lie algebra of Aff(1) is Frobenius. Other examples are parabolic subgroups which are Fobenius as well (see [7] and[8]). But we thought that Grossmann's work is the best example for young researchers how to understand the square-integrable representations for case nonunimodular groups which is started from the Aff(1) Lie group. Moreover, other examples of nonunimodular groups are Lie groups whose Lie algebras are 4-dimensional real Frobenius Lie algebras. Kurniadi and Ishi [9] showed that irreducible unitary representations of these Lie groups are square-integrable representations and they wrote the Duflo-Moore operators in the terms of groups Fourier transforms.

Many reseachers study affine Lie algebras and the structure of affine for instance we see some results in [10], [11], [12], [13],[14], [15], [16], and [17].

In the other hand, in easier stage we can also study square-integrable representations for unimodular Lie groups case. Heisenberg Lie groups of dimension $2 n+1$ and filiform Lie groups are in these types. In fact, the Duflo-Moore operators for square-integrable representations of unimodular groups are scalar multiple (see [18]). In current work, Kurniadi in [19] proved that irreducible-unitary representation of Lie group of 4dimensional standard filiform Lie algebra is square-integrable and its Duflo-Moore operator is scalar multiple of identity which is equal to one.

In this work, we shall give another alternative to compute the Duflo-Moore operator for square-integrable representation of $\mathrm{Aff}^{+}(1)$ by direct computations instead of forming in group Fourier transform which was written in [18].

\section{Preliminaries}

Let $\mathrm{Aff}^{+}(1)$ be the 2-dimensional affine Lie group whis is expressed as a semidirect product of the set of all real numbers $\mathbb{R}$ and the set of all positive real numbers $\mathbb{R}_{+}$. Namely, we can write this group as $\operatorname{Aff}^{+}(1):=\mathbb{R} \rtimes \mathbb{R}_{+}$. Particularly, in this work we concentrate to $\mathrm{Aff}^{+}(1)$ which is the exponential solvable nonunimodular Lie group. To make easier in computations we write $\mathrm{Aff}^{+}(1)$ in matrix terms. Namely, we have

$$
\operatorname{Aff}^{+}(1) \ni\left(\begin{array}{cc}
\alpha & \beta \\
0 & 1
\end{array}\right), \alpha \in \mathbb{R}_{+}, \beta \in \mathbb{R}
$$

Regarding this notations, we denote $g(\alpha, \beta):=\left(\begin{array}{cc}\alpha & \beta \\ 0 & 1\end{array}\right), \Delta(\alpha):=\left(\begin{array}{ll}\alpha & 0 \\ 0 & 1\end{array}\right)$, and $\nabla(\beta):=$ $\left(\begin{array}{ll}1 & \beta \\ 0 & 1\end{array}\right)$. The Lie algebra of $\operatorname{Aff}^{+}(1)$ is denoted by aff(1) whose basis is $\left\{e_{1}, e_{2}\right\}$. The nonzero bracket of aff(1) is given by $\left[e_{1}, e_{2}\right]=e_{2}$. The Lie algebra aff(1) is a Frobenius Lie algebra which has two open coadjoint orbits as follows (see [20]).

$$
\Omega_{ \pm}:=\{(a, b) \quad ; \quad a, b \in \mathbb{R}, \pm b>0\} .
$$


The representations of the affine Lie group Aff(1) can be realized on the Hilbert space of all square-integrable functions $L^{2}\left(\mathbb{R}_{+}\right)$. Before doing that, let us mention here some basic notion of representation theory of Lie groups corresponding to our research.

Definition 1 [21]. Let $\pi$ be a representation of a Lie group $G$ on the carrier space $\mathcal{H}$. $\pi$ is said to be irreducible if $\pi$ has no nontrivial $\pi$-invariant subspace $\mathcal{H}_{0}$ in $\mathcal{H}$. Moreover, $\pi$ is said to be uintary if for each $\mathrm{f} \in \mathcal{H}$ and each $\mathrm{g} \in \mathrm{G}$

$$
\|\pi(g) f\|=\|f\| .
$$

Proposition 2 [20]. The irreducible unitary representations of $\mathrm{Aff}^{+}(1)$ correponsding to open coadjoint orbit $\Omega_{+}$in eqs. (2) in the space $L^{2}\left(\mathbb{R}_{+}\right)$is of the form

$$
\pi_{+}(g) f(x)=e^{2 \pi i \beta x} f(\alpha x)
$$

where $g:=g(\alpha, \beta) \in \operatorname{Aff}^{+}(1), \alpha \in \mathbb{R}_{+}, \beta \in \mathbb{R}$ and $f \in \mathrm{L}^{2}\left(\mathbb{R}_{+}\right)$.

Furthermore, the representation of affine Lie group $\mathrm{Aff}^{+}(1)$ can be realized as a quasi-regular representations (see [18]). It is written in the formula as follows.

$$
\pi(g(\alpha, \beta))=\alpha^{-\frac{1}{2}} \psi\left(\frac{x-\beta}{\alpha}\right), \alpha \in \mathbb{R}_{+}, \beta \in \mathbb{R} \text { and } \psi \in \mathrm{L}^{2}\left(\mathbb{R}_{+}\right) .
$$

We are mainly interested in the square-integrable representation. Let $\pi$ be an irreducible unitary representation of a Lie group $G$ realized on the space $\mathcal{H}$ and $\mathrm{L}^{2}(G)$ be the space of all square-integrable functions on $G$. For vector $f_{1} \in \mathcal{H}$, we define the operator on $\mathcal{H}$ given by

$$
\mathcal{E}_{f_{1}}: \mathcal{H} \ni f_{2} \mapsto \mathcal{E}_{f_{1}} f_{2} \in \mathrm{L}^{2}(G) .
$$

where $\varepsilon_{f_{1}} f_{2}(x)=\left\langle f_{1} \mid \pi(x) f_{2}\right\rangle$.

Definition 3 [22].The irreducible unitary representation $\pi$ of locally compact topological group $\mathrm{G}$ realized on a space $\mathcal{H}$ is said to be square-interable if there exist two vectors $\mathrm{f}_{1}, \mathrm{f}_{2} \in \mathcal{H}-\{0\}$ such that

$$
\left\|\varepsilon_{f_{1}} f_{2}\right\|^{2}=\left\langle f_{1} \mid \pi(x) f_{2}\right\rangle=\int_{G} f_{1}(g) \overline{\pi(x) f_{2}(g)} \mathrm{d} \mu(g)<+\infty .
$$

In the other words, $\left\langle\mathrm{f}_{1} \mid \pi(\mathrm{x}) \mathrm{f}_{2}\right\rangle \in \mathrm{L}^{2}\left(\mathrm{G}, \mu_{\mathrm{G}}\right)$ where $\mu_{\mathrm{G}}$ is a measure on $\mathrm{G}$. Such vectors which satisfied eqs. (7) are called admissible vectors.

Duflo-Moore state their results in the following theorem

Theorem 4 [23]. If $\pi$ is square-integrable representations of locally compact group $G$ realized on the space $\mathcal{H}$ then there exists a positive selfadjoint operator $\mathrm{C}_{\pi}: \mathcal{H} \rightarrow \mathcal{H}$ which is called the Duflo-Moore operator such that

a. a vector $\psi \in \mathcal{H}-\{0\}$ is admissible if and only if $\psi$ is an element of domain of $\mathrm{C}_{\pi}$.

b. if $\mathrm{f}_{1}, \mathrm{f}_{2} \in \mathcal{H}$ and $\mathrm{f}_{3}, \mathrm{f}_{4} \in \operatorname{Dom}\left(\mathrm{C}_{\pi}\right)$ then

$$
\left\langle\varepsilon_{f_{1}} f_{3} \mid \mathcal{E}_{f_{2}} f_{4}\right\rangle_{\mathrm{L}^{2}\left(G, \mu_{G}\right)}=\left\langle f_{1} \mid f_{2}\right\rangle_{\mathcal{H}}\left\langle C_{\pi} f_{4} \mid C_{\pi} f_{3}\right\rangle_{\mathcal{H}}
$$




\section{Methods}

In this research we apply the literature reviews method, particularly we focus on results in [18] and [20]. We obtain the quasi-regular representation of $\mathrm{Aff}^{+}(1)$ in Fuhr's work and we compute the Fourier transform of its representation to determine the DufloMoore operator. On the other hand, we also obtain the irreducible unitary representation of $\mathrm{Aff}^{+}(1)$ corressponding to open coadjoint orbits and we show that representatation is square-integrable. Using direct computations, we obtain the Duflo-Moore operator for that representation.

\section{Results and Discussion}

Our results and discussion consist of two main part as follows.

\subsection{The Duflo-Moore Operator for The Quasi-Regular Representation of $\operatorname{Aff}^{+}(1)$.}

The following statement can be deduced from [18] in page 30--31. However, we give a detail proof for its own interest.

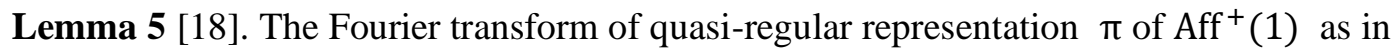
eqs. (5) is of the form

$$
\mathcal{F}(\pi(g(\alpha, \beta)) \psi)(\xi)=\alpha^{\frac{1}{2}} e^{-2 \pi i \xi \beta} \mathcal{F} \psi(\alpha \xi) .
$$

\section{Proof.}

By direct computation we obtain

$$
\begin{aligned}
\mathcal{F}(\pi(g(\alpha, \beta)) \psi)(\xi) & =\int_{\mathbb{R}} e^{-2 \pi i \xi x}(\pi(g(\alpha, \beta)) \psi)(x) d x \\
& =\int_{\mathbb{R}} e^{-2 \pi i \xi x} \alpha^{-1 / 2} \psi\left(\frac{x-\beta}{\alpha}\right) d x \\
& =\int_{\mathbb{R}} e^{-2 \pi i \xi(\alpha \eta+\beta)} \alpha^{-1 / 2} \psi(\eta) \alpha d \eta \\
& =\int_{\mathbb{R}} e^{-2 \pi i \xi(\alpha \eta)} e^{-2 \pi i \xi \beta} \alpha^{1 / 2} \psi(\eta) d \eta \\
& =\int_{\mathbb{R}} e^{-2 \pi i(\alpha \xi) \eta} e^{-2 \pi i \xi \beta} \alpha^{1 / 2} \psi(\eta) d \eta \\
& =e^{-2 \pi i \xi \beta} \alpha^{1 / 2} \int_{\mathbb{R}} e^{-2 \pi i(\alpha \xi) \eta} \psi(\eta) d \eta \\
& =e^{-2 \pi i \xi \beta} \alpha^{\frac{1}{2} \mathcal{F} \psi(\alpha \xi) .}
\end{aligned}
$$

Proposition 6 [18]. The Duflo-Moore operator for quasi-regular representation $\pi$ of $\mathrm{Aff}^{+}(1)$ as in eqs. (5) in the term of Fourier transform can be written as follows. 


$$
\mathcal{F}\left(C_{\pi} \psi\right)(\xi)=\xi^{-1 / 2} \mathcal{F} \psi(\xi)
$$

Proof. Let $\psi_{1}$ and $\psi_{2}$ be elements of contiunuous functions space of compact support on $\mathrm{Aff}^{+}(1)$ denoted by $C_{c}\left(\mathrm{Aff}^{+}(1)\right)$. Using Plancherel's theorem and Fubini's theorem we obtain

$$
\begin{aligned}
& \int_{\operatorname{Aff}^{+}(1)}\left|\left\langle\psi_{1} \mid \pi(g(\alpha, \beta)) \psi_{2}\right\rangle\right|^{2} \frac{d \alpha}{\alpha^{2}} d \beta=\int_{\operatorname{Aff}^{+}(1)}\left|\left\langle\mathcal{F} \psi_{1} \mid \mathcal{F} \pi(g(\alpha, \beta)) \psi_{2}\right\rangle\right|^{2} \frac{d \alpha}{\alpha^{2}} d \beta \\
& =\int_{\operatorname{Aff}^{+}(1)}\left|\int_{\mathbb{R}} \mathcal{F} \psi_{1}(\xi) \overline{e^{-2 \pi l \xi \beta} \alpha^{1 / 2} \mathcal{F} \psi}(\alpha \xi) d \xi\right|^{2} \frac{d \alpha}{\alpha^{2}} d \beta \\
& =\int_{\operatorname{Aff}^{+}(1)}\left|\int_{\mathbb{R}} \mathcal{F} \psi_{1}(\xi) e^{2 \pi i \xi \beta} \alpha^{1 / 2} \overline{\mathcal{F} \psi}(\alpha \xi) d \xi\right|^{2} \frac{d \alpha}{\alpha^{2}} d \beta \\
& =\int_{\mathbb{R}^{\prime}} \int_{\mathbb{R}_{+}}\left|\int_{\mathbb{R}} \mathcal{F} \psi_{1}(\xi) e^{2 \pi i \xi \beta} \overline{\mathcal{F} \psi}(\alpha \xi) d \xi\right|^{2} \frac{d \alpha}{\alpha} d \beta \\
& =\int_{\mathbb{R}} \int_{\mathbb{R}_{+}}\left|\mathcal{F} \tau_{\alpha}(-\beta)\right|^{2} \frac{d \alpha}{\alpha} d \beta \\
& \left(\tau_{\alpha}(\xi)=\mathcal{F} \psi_{1}(\xi) \overline{\mathcal{F} \psi}(\alpha \xi)\right) \\
& =\int_{\mathbb{R}} \int_{\mathbb{R}_{+}}\left|\mathcal{F} \psi_{1}(\xi) \overline{\mathcal{F} \psi}(\alpha \xi)\right|^{2} \frac{d \alpha}{\alpha} d \beta \\
& =\int_{\mathbb{R}}\left|\mathcal{F} \psi_{1}(\xi)\right|^{2}\left\{\int_{\mathbb{R}_{+}}|\overline{\mathcal{F} \psi}(\alpha \xi)|^{2} \frac{d \alpha}{\alpha}\right\} d \xi \\
& =\left\{\int_{\mathbb{R}}\left|\mathcal{F} \psi_{1}(\xi)\right|^{2} d \xi\right\}\left\{\int_{\mathbb{R}_{+}}|\overline{\mathcal{F} \psi}(\alpha \xi)|^{2} \frac{d \alpha}{\alpha}\right\} \\
& =\left\{\int_{\mathbb{R}}\left|\mathcal{F} \psi_{1}(\xi)\right|^{2} d \xi\right\}\left\{\int_{\mathbb{R}}\left|\overline{\mathcal{F} \psi}\left(\alpha^{\prime}\right)\right|^{2} \frac{d \alpha^{\prime}}{\alpha^{\prime}}\right\} \\
& \left(\alpha^{\prime}:=\alpha \xi\right) \text {. } \\
& =\left\|\mathcal{F} \psi_{1}\right\|^{2}\left\{\int_{\mathbb{R}}\left|\overline{\mathcal{F} \psi}\left(\alpha^{\prime}\right)\right|^{2} \frac{d \alpha^{\prime}}{\alpha^{\prime}}\right\} .
\end{aligned}
$$

Thus, from the latter equation we obtain the Duflo-Moore operator is equal to $\mathcal{F}\left(C_{\pi} \psi\right)(\xi)=\xi^{-1 / 2} \mathcal{F} \psi(\xi)$ as desired.

\subsection{The Duflo-Moore Operator for The Irreducible Unitary Representation of $\operatorname{Aff}^{+}(1)$}

This session is the main result. First, we recall that the irreducible unitary representation of group $\mathrm{Aff}^{+}(1)$ in Proposition 2 can be written in the following proposition 
Proposition 7. The irreducible unitary representations of $\mathrm{Aff}^{+}(1)$ correponsding to open coadjoint orbit $\Omega_{+}$in eqs. (2) in the space $L^{2}\left(\mathbb{R}_{+}\right)$is of the form

$$
\begin{gathered}
\pi_{+}(\nabla(\beta)) f(x)=e^{2 \pi i \beta x} f(x),
\end{gathered}
$$

where $\alpha \in \mathbb{R}_{+}, \beta \in \mathbb{R}$ and $f \in \mathrm{L}^{2}\left(\mathbb{R}_{+}\right)$.

Proof. Let aff(1) be a Lie algebra of $\operatorname{Aff}^{+}(1)$ whose basis is $\left\{e_{1}, e_{2}\right\}$. We consider its dual space as $\operatorname{aff}(1)^{*} \ni\left(\begin{array}{ll}a & * \\ b & *\end{array}\right)$, where $a, b \in \mathbb{R}$. Moreover, let $\left(\begin{array}{ll}\alpha & \beta \\ 0 & 1\end{array}\right), \alpha \in \mathbb{R}_{+}, \beta \in$ $\mathbb{R}$ be an element of group affine $\mathrm{Aff}^{+}(1)$. We shall construct the irreducible unitary representation of $\mathrm{Aff}^{+}(1)$ corresponding to open coadjoint orbit $\Omega_{+}:=\{(a, b) ; b>$ $0\}$. To do that, fix a point $\tau:=e_{2}^{*} \in \Omega_{+} \subset$ aff(1) ${ }^{*}$ as a linear functional. For subalgebra $\aleph:=\left\langle e_{2}\right\rangle$ we have $\aleph$ has maximal dimension and the value of linear functional $\tau$ on the commutator $[\aleph, \aleph]$ is given by $\tau([\aleph, \aleph])=0$. Therefore, $\aleph$ is a polarization in aff(1). Let $\aleph^{\perp}$ be the orthogonal subspace. Furthermore, since $\tau+\aleph^{\perp}$ is contained in $\Omega_{+}$then $\boldsymbol{N}$ satisfies Pukanszky condition.

Now we construct a 1-dimensional representation $\lambda_{\tau}$ of $N:=\exp N$ as follows.

$$
\lambda_{\tau}(\exp e):=e^{2 \pi i\langle\tau \mid e\rangle}=e^{2 \pi i \beta}, e:=\alpha e_{1}+\beta e_{2}, \tau \in \Omega_{+} .
$$

We identify the coset $\mathrm{Aff}^{+}(1) / \mathrm{N}$ by $\mathbb{R}_{+}$and we obtain the section given by

$$
s: \mathbb{R}_{+} \ni x \mapsto \exp x e_{1} \in \operatorname{Aff}^{+}(1) .
$$

To obtain the explicit formula of the representation of $\mathrm{Aff}^{+}(1)$ we need to solve what we called the master equation

$$
s(x) g=h_{s}(x, g) s(x g), \quad\left(x \in \mathbb{R}_{+}, g \in \mathrm{Aff}^{+}(1), h_{s}(x, g) \in \mathrm{N}\right) .
$$

Using the basis $\left\{e_{1}, e_{2}\right\}$ we solve the following master equations with respect to its basis:

a. $\left(\begin{array}{ll}x & 0 \\ 0 & 1\end{array}\right)\left(\begin{array}{ll}\alpha & 0 \\ 0 & 1\end{array}\right)=\left(\begin{array}{ll}1 & u \\ 0 & 1\end{array}\right)\left(\begin{array}{ll}y & 0 \\ 0 & 1\end{array}\right)$,

by solving with respect to $u$ and $y$ we obtain $y=\alpha x$. Therefore, $\pi_{+}(\Delta(\alpha)) f(x)=$ $f(\alpha x)$. We mention here that we apply a right action of $\operatorname{Aff}^{+}(1)$ in space $\mathrm{L}^{2}\left(\mathbb{R}_{+}\right)$.

b. $\left(\begin{array}{ll}x & 0 \\ 0 & 1\end{array}\right)\left(\begin{array}{ll}1 & \beta \\ 0 & 1\end{array}\right)=\left(\begin{array}{ll}1 & u \\ 0 & 1\end{array}\right)\left(\begin{array}{ll}y & 0 \\ 0 & 1\end{array}\right)$.

In this case, we have $y=x$ and $u=\beta x$. Therefore, $\pi_{+}(\nabla(\beta)) f(x)=e^{2 \pi i \beta x} f(x)$ as desired.

In the next section, we shall compute the Duflo-Moore operator for the representation of $\mathrm{Aff}^{+}(1)$ with respect to its right Haar measure. The result of DufloMoore operator for the representation of $\mathrm{Aff}^{+}(1)$ with respect left Haar measure can be found in [24] pages 82-85. 
Proposition 8. The Duflo-Moore operator for the irreducible unitary representation $\pi_{+}$of $\mathrm{Aff}^{+}(1)$ as written in eqs. (11) is of the form

$$
C_{\pi_{+}} f(\Delta(x))=x^{-1 / 2} f(x), \quad\left(f \in \mathrm{L}^{2}\left(\mathbb{R}_{+}\right), x \in \mathbb{R}_{+}\right)
$$

Proof. Let $\vartheta_{1}$ and $\vartheta_{2}$ be elements in $C_{c}\left(\operatorname{Aff}^{+}(1)\right)$. Using the right Haar measure, we shall compute the integral

$$
\int_{\mathrm{Aff}^{+}(1)}\left|\left\langle\vartheta_{1} \mid \pi_{+}(\nabla(\beta)) \pi_{+}(\Delta(\alpha)) \vartheta_{2}\right\rangle_{\mathrm{L}^{2}\left(\mathbb{R}_{+}\right)}\right|^{2} d \beta \frac{d \alpha}{\alpha}
$$

To do that, first we compute the following inner product.

$$
\begin{aligned}
\left\langle\vartheta_{1} \mid \pi_{+}(\nabla(\beta)) \pi_{+}(\Delta(\alpha)) \vartheta_{2}\right\rangle_{L^{2}\left(\mathbb{R}_{+}\right)} & =\int_{\mathbb{R}_{+}} \vartheta_{1}(x) \overline{\pi_{+}(\nabla(\beta)) \pi_{+}(\Delta(\alpha)) \vartheta_{2}}(x) \frac{d x}{x} \\
& =\int_{\mathbb{R}_{+}} \vartheta_{1}(x) \overline{\pi_{+}(\Delta(\alpha)) e^{2 \pi l \beta x} \vartheta_{2}}(x) \frac{d x}{x} \\
& =\int_{\mathbb{R}_{+}} e^{-2 \pi i \beta x} \vartheta_{1}(x) \overline{\pi_{+}(\Delta(\alpha)) \vartheta_{2}}(x) \frac{d x}{x} .
\end{aligned}
$$

Using Plancherel's theorem we have

$$
\begin{aligned}
\int_{\mathbb{R}}\left|\left\langle\vartheta_{1} \mid \pi_{+}(\nabla(\beta)) \pi_{+}(\Delta(\alpha)) \vartheta_{2}\right\rangle_{\mathrm{L}^{2}\left(\mathbb{R}_{+}\right)}\right|^{2} d \beta & =\int_{\mathbb{R}_{+}}\left|e^{-2 \pi i \beta x} \vartheta_{1}(x) \overline{\pi_{+}(\Delta(\alpha)) \vartheta_{2}}(x)\right|^{2} \frac{d x}{x^{2}} \\
& =\int_{\mathbb{R}_{+}}\left|\vartheta_{1}(x) \overline{\pi_{+}(\Delta(\alpha)) \vartheta_{2}}(x)\right|^{2} \frac{d x}{x^{2}} \\
& =\int_{\mathbb{R}_{+}}\left|\vartheta_{1}(x) \overline{\vartheta_{2}}(\alpha x)\right|^{2} \frac{d x}{x^{2}} .
\end{aligned}
$$

Therefore, using Fubini's theorem we obtain

$$
\begin{aligned}
\int_{\operatorname{Aff}^{+}(1)}\left|\left\langle\vartheta_{1} \mid \pi_{+}(\nabla(\beta)) \pi_{+}(\Delta(\alpha)) \vartheta_{2}\right\rangle_{L^{2}\left(\mathbb{R}_{+}\right)}\right|^{2} d \beta \frac{d \alpha}{\alpha}=\int_{\mathbb{R}_{+}}\left|\vartheta_{1}(x)\right|^{2}\left\{\int_{\mathbb{R}_{+}}\left|\vartheta_{2}(\alpha x)\right|^{2} \frac{d \alpha}{\alpha}\right\} \frac{d x}{x^{2}} \\
=\int_{\mathbb{R}_{+}}\left|\vartheta_{1}(x)\right|^{2} \frac{d x}{x^{2}}\left\{\int_{\mathbb{R}_{+}}\left|\vartheta_{2}\left(\alpha^{\prime}\right)\right|^{2} \frac{d \alpha^{\prime}}{\alpha^{\prime}}\right\} \\
\left.=\int_{\mathbb{R}_{+}} \mid x^{\prime}:=\alpha x\right) \\
=\int_{\mathbb{R}_{+}}\left|x^{-1 / 2} \vartheta_{1}(x)\right|^{2} \frac{d x}{x}\left\{\int_{\mathbb{R}_{+}}\left|\vartheta_{2}\left(\alpha^{\prime}\right)\right|^{2} \frac{d \alpha^{\prime}}{\alpha^{\prime}}\right\}
\end{aligned}
$$


Therefore, The Duflo-Moore operator for the irreducible unitary representation of $\mathrm{Aff}^{+}(1)$ as written in eqs. (11) is of the form $C_{\pi_{+}} f(\Delta(x))=x^{-1 / 2} f(x)$ as desired.

\section{Conclusions}

The Duflo-Moore operator for the representations of $\mathrm{Aff}^{+}(1)$ in this paper is considered in two cases. The first case, it is for the quasi-regular representation and written in the term of Fourier transform. Namely, we obtain $\mathcal{F}\left(C_{\pi} \psi\right)(\xi)=\xi^{-1 / 2} \mathcal{F} \psi(\xi)$ (see [18]). The second case, the Duflo-Moore operator is considered for irreducible unitary representation with respect to its right Haar measure and we have $C_{\pi_{+}} f(\Delta(x))=$ $x^{-1 / 2} f(x)$. On the other hand, the Duflo-Moore operator for a square-intergarble representation of $\mathrm{Aff}^{+}(1)$ with respect to its left Haar measure can be seen in [24] pages $82-85$.

It is more interesting to compute the Duflo-Moore operator for the representation of higher dimension of affine Lie groups.

\section{Acknowledgement}

The first author is very grateful to Professor Hideyuki Ishi from Osaka City University Japan for generous support and guidance to the first author to study representation theory of Lie groups during his study. We also thank to the University of Padjadjaran who has funded the work through Riset Percepatan Lektor Kepala (RPLK) year 2020 with contract number 1427/UN6.3.1/LT/2020.

\section{References}

[1] K. Grochenig and D. Rottensteiner, "Orthonormal bases in the orbit of squareintegrable representations of nilpoten Lie groups," J. Funct. Anal., vol. 275, no. 12, pp. 3338--3379, 2018.

[2] A. . Farashahi, "Square-integrability of metaplectic wave-packet representations of L^2(R)," J. Math. Anal. Appl., vol. 449, no. 1, 2017.

[3] A. Grossmann, J. Morlet, and T. Paul, "Transform associated to square-integrable group of representations I," J.Math.Phys, vol. 26, pp. 2473--2479, 1985.

[4] A. Grossmann, J. Morlet, and T. Paul, "Trsansform associated to square-integrable group representations .II. Examples," Ann.Inst.H.Poincare Phys.Theor, vol. 45, pp. 293--309, 1986.

[5] P. Stachura, "On the quantum $a x+b$ group," J. Geom. Phys., vol. 73, pp. 125-149, 2013.

[6] Zeitlin,A.M, "Unitary representations of a loop ax+b group, Wiener measure and IGamma -function," J. Funct. Anal., vol. 263, no. 3, pp. 529--548, 2012.

[7] M. . Dyer and G. . Lehres, "Parabolic subgroup orbits on finite root systems," $J$. Pure Appl. Algebr., vol. 222, no. 12, pp. 3849--3857, 2018.

[8] M. Calvez and et al, "Conjugacy stability of parabolic subgroups of Artin-Tits groups of spherical type," J. Algebr., vol. 556, pp. 621--633, 2020.

[9] E. Kurniadi and H. Ishi, "Harmonic analysis for 4-dimensional real Frobenius Lie algebras," in Springer Proceeding in Mathematics \& Statistics, 2019.

[10] W. Rump, "Affine stucture of decomposable solvable groups," J. Algebr., vol. 556, pp. 725--749, 2020. 
[11] H. Li and Q. Wang, "Trigonometric Lie algebras, affine Lie algebras, and vertex algebras," J. Adv. Math., vol. 363, 2020.

[12] J. . Souza, "Sufficient conditions for dispersiveness of invariant control affine system on the Heisenberg group," Syst. \&Control Lett., vol. 124, pp. 68--74, 2019.

[13] E. Marberg, "On some actions the 0-zero hecke monoids of affine symmetric groups," J. Comb. Theory, vol. 161, pp. 178--219, 2019.

[14] Ayala,V, A. Da Silva, and M. Ferreira, "Affine and bilinear systems on Lie groups," Syst. \&Control Lett., vol. 117, pp. 23--29, 2018.

[15] F. Catino, I. Colazzo, and P. Stefanella, "Regular subgroups of the affine group and asymmetric product of radical braces," J. Algebr., vol. 455, pp. 164--182, 2016.

[16] D. Burde and et al, "Affine actions on Lie groups and post-Lie algebra structures," J. Linear Algebr. Its Appl., vol. 437, no. 5, pp. 1250--1263, 2012.

[17] H. Kato, "Low dimensional Lie groups admitting left-invariant flat projective or affine structures," J. Differ. Geom. Its Appl., vol. 30, no. 2, pp. 153--163, 2012.

[18] H. Fuhr, Abstrac harmonic analysis of continuous wavelet transforms, Lecture notes in mathematics. Berlin: Springer-Verlag, 2005.

[19] E. Kurniadi, "On Square-Integrable Representations of A Lie Group of 4Dimensional Standard Filiform Lie Algebra," CauchyJurnal Mat. Murni dan Apl., 2020.

[20] A. A. Kirillov, "Lectures on the Orbit Method, Graduate Studies in Mathematics," Am. Math. Soc., vol. 64, 2004.

[21] R. Berndt, Representation of linear groups. An introduction based on examples from physics and number theory. Wiesbaden: Vieweg, 2007.

[22] P. Aniello, G. Cassinelli, E. de Vito, and Levrero,A., "Square-integrability of induced representations of semidirect products," Rev.Math.Phys, vol. 10, pp. 301-313, 1998.

[23] M. Duflo and C. C. Moore, "On the Regular Representation of a nonunimodular Locally Compact," J. Funct. Anal., vol. 21, pp. 209-243, 1976.

[24] E. Kurniadi, "Harmonic analysis for finite dimensional real Frobenius Lie algebras, Ph.D thesis, " Nagoya University, 2019. 